\title{
DIMENSÕES DO PROCESSO DE FORMAÇÃO DE ESTRATÉGIA EM PEQUENOS HOTÉIS DE CURITIBA ${ }^{1}$
}

\author{
Dimensions in the process of strategy development \\ in small hotels in Curitiba
}

\begin{abstract}
Daniela Torres da Rocha ${ }^{[a]}$, Sara Culti Gimenez ${ }^{[b]}$, Fernando Antonio Prado Gimenez ${ }^{[c]}$
[a] Administradora, Mestranda em Administração Programa de Pós-Graduação em Administração da Pontifícia Universidade Católica do Paraná (PPAD/PUCPR), Curitiba, PR - Brasil, e-mail: danitorres.rocha@gmail.com

[b] Aluna do curso de Graduação em Turismo, Universidade Positivo (UP), bolsista de iniciação científica CNPq, Curitiba, PR Brasil, e-mail: sara.gimenez@hotmail.com

[c] Administrador, Doutor, professor do Programa de Pós-Graduação em Administração da Pontifícia Universidade Católica do Paraná (PPAD/PUCPR), Curitiba, PR - Brasil, e-mail: fernando.gimenez@pucpr.br
\end{abstract}

\section{Resumo}

Uma questão relevante, tanto do ponto de vista teórico, quanto do ponto de vista prático, é entender quais são os principais fatores que influenciam o processo de formação de estratégia em pequenos hotéis. $\mathrm{O}$ trabalho buscou identificar as dimensões predominantes no processo de desenvolvimento de estratégia em pequenos hotéis. A coleta de dados foi feita em uma amostra não aleatória de 18 pequenos hotéis localizados na cidade de Curitiba, PR. Os dados foram coletados por meio da aplicação de um questionário, em contato pessoal com o principal dirigente de cada empresa visitada. Nesse instrumento de coleta de dados havia dois conjuntos de questões. O primeiro bloco levantou informações demográficas sobre a empresa e seu dirigente. O segundo bloco foi composto pelo Questionário de Desenvolvimento de Estratégia (QDE) proposto por Bailey e Avery (1998). Os resultados revelaram que nas pequenas empresas estudadas há a predominância do incrementalismo, ou seja, processos emergentes de formação de estratégia, combinado com aspectos de planejamento e culturais.

Palavras-chave: Estratégia. Pequenos hotéis. Processo estratégico.

\section{Abstract}

A relevant question, from the theoretical point of view as well as from the practical one, is to understand which are the main factors that affect the process of strategy formation in small hotels. The paper aimed to identify

\footnotetext{
1 Este trabalho foi originalmente apresentado no XI Seminário Internacional de Turismo. Curitiba: OBSTUR/UFPR; UP, 2009.
} 
the predominant dimensions in the process of strategy development in small hotels. Data collection was made through personal contact with owner-managers using a structured questionnaire. The instrument had two blocks of questions. The first gathered demographical information on the firms and respondents. The second was composed by Strategy Development Questionnaire (SDQ) proposed by Bailey and Avery (1998). Results indicated that there is a predominance of incrementalism, i. e., emergent processes in the strategy formation, combined with planning and cultural factors..

Keywords: Strategy. Small hotels. Strategic process.

\section{INTRODUÇÃO}

Cooper (1981), em um trabalho seminal, examinou os fatores que influenciam a formulação e implantação de estratégias em empresas novas e pequenas. Argumentando que o contexto das pequenas empresas é diverso do das grandes empresas, este autor analisou a formulação e implantação de estratégia com base em duas características principais das pequenas empresas: o tipo de pequena empresa e o estágio no ciclo de vida.

As pequenas empresas variam em diversas dimensões, tais como disponibilidade de recursos, experiência anterior dos fundadores, sofisticação administrativa e desempenho. Estas diferenças podem ser representadas por arquétipos que foram denominadas por Cooper (1981), assim:

- empresas de casais (mom and pop companies), que, em geral, têm poucos empregados ou até mesmo nenhum, sistemas administrativos pouco desenvolvidos, proprietários com habilidades técnicas, mas pouco conhecimento gerencial, e baixo retorno aos proprietários, que podem voltar a ser empregados em outras empresas nos momentos de crise; - empresas estáveis de alto retorno (stable highpay-off companies), que possuem sistemas administrativos mais formalizados, desfrutam de vantagens competitivas mais sólidas, com um desempenho mais estável, fornecendo um padrão de vida adequado aos seus proprietários;

- empresas orientadas para o crescimento (growth-oriented companies), que são, em geral, iniciadas porgrupos, seus administradores têm maior experiência gerencial e educação formal, enfatizam a inovação constante e estão posicionadas em mercados crescentes.

No que diz respeito ao estágio de desenvolvimento das pequenas empresas, Cooper (1981) distingue três períodos. O primeiro é o de criação da empresa (start-up), que envolve as decisões estratégicas de fundar uma empresa e posicioná-la em uma indústria com uma estratégia competitiva específica. O segundo estágio, denominado de crescimento inicial (early-growth), é o período em que a estratégia inicial de produtos e mercados está sendo testada e o empreendedor se envolve diretamente em todas as atividades da empresa. Para Cooper (1981), muitas pequenas empresas se estabilizam nesse estágio. O terceiro estágio é o de crescimento tardio (later-growth), que se caracteriza pela existência de múltiplos locais de operação para empresas varejistas e de serviços ou diversificação de produção para empresas industriais. Nesse estágio, a estrutura administrativa é mais complexa, com um ou mais níveis médios de gerenciamento.

A combinação do tipo de empresa e estágio de desenvolvimento coloca para os proprietários de pequenas empresas diferentes necessidades e oportunidades estratégicas que impactam na formulação e implantação de estratégias competitivas.

No campo da administração estratégica, estudos mais recentes têm demonstrado que o processo de formação e implantação de estratégias competitivas é complexo, sofrendo influência de diversos tipos, tais como a experiência e a história de vida dos estrategistas, modelos mentais dos principais dirigentes, dinâmica do espaço competitivo, capacidade de aprendizagem organizacional, crenças e valores predominantes no âmbito da empresa, entre 
outros (MINTZBERG; QUINN, 2001; JENKINS; AMBROSINI, 2002).

No mercado hoteleiro de Curitiba encontram-se muitos pequenos hotéis independentes, alguns com décadas de existência, que têm enfrentado a crescente competição de redes hoteleiras. A presença dessas pequenas organizações nesse mercado extremamente competitivo (HOCAYENDA-SILVA; TEIXEIRA, 2007) deve-se à capacidade que seus administradores possuem de ajustar a estratégia adotada a condições ambientais mutáveis. Uma questão relevante, tanto do ponto de vista teórico quanto do ponto de vista prático, é entender quais são os principais fatores que influenciam o processo de formação de estratégia em pequenos hotéis. Do ponto de vista teórico, responder a esta questão pode trazer novos conhecimentos sobre este fenômeno que foi alvo das reflexões de Cooper há quase três décadas e tem tido uma explicação ampliada por outros estudiosos do tema. Do ponto de vista prático, o entendimento dos fatores que influenciam a formação e implantação de estratégia em pequenos hotéis pode ajudar seus dirigentes a perceberem espaços de decisão que não têm merecido a devida atenção no gerenciamento de pequenos hotéis.

Dessa forma, o propósito deste artigo é apresentar os resultados de uma pesquisa feita com dirigentes de pequenos hotéis independentes localizados na cidade de Curitiba. A investigação buscou identificar as dimensões predominantes no processo de desenvolvimento de estratégia em pequenos hotéis, conforme modelo proposto por Bailey e Avery (1998).

O trabalho encontra-se estruturado em quatro seções adicionais a esta introdução. $\mathrm{Na}$ próxima seção, são apresentadas as dimensões que influenciam no processo de desenvolvimento de estratégia segundo Bailey e Avery (1998). Nesta seção, ainda, comenta-se os resultados de trabalhos anteriores que trataram da questão da formação de estratégia em pequenas empresas. A terceira seção é dedicada à apresentação dos procedimentos de pesquisa adotados neste estudo e comenta-se sobre suas limitações. Na quarta seção são apresentados os resultados do estudo, por meio da descrição de características demográficas das empresas e dirigentes pesquisados e das dimensões predominantes na formação de estratégia desses hotéis. Por fim, na conclusão, reflete-se sobre o aprendizado obtido por meio deste estudo.

\section{FORMAÇÃO DE ESTRATÉGIA EM PEQUENAS EMPRESAS}

O tema da formação de estratégia tem sido abordado de forma crescente na literatura administrativa. Muitas são as perspectivas e os enfoques teóricos que norteiam os estudos da área (MINTZBERG; AHLSTRAND; LAMPEL, 2000; HIT'T et al., 2002). De igual forma, encontra-se na literatura uma crescente preocupação com a análise desse fenômeno no âmbito de empresas de pequeno porte (ROBINSON Jr.; PEARCE II, 1984; D'AMBOISE; MULDOWNEY, 1988). O objetivo dessa seção é apresentar um modelo conceitual proposto em 1998 por Andy Bailey e Clare Avery sobre as diferentes configurações de processos de formação de estratégia, ao mesmo tempo em que são descritos estudos recentes publicados no Brasil e no exterior que abordaram esse processo em pequenas empresas.

Mintzberg, Ahsltrand e Lampel (2000) apresentaram dez escolas de pensamento no campo da administração estratégica. A Escola do Design, a Escola do Planejamento e a Escola do Posicionamento propõem que a formação da estratégia é um processo consciente e deliberado de alinhar a organização com seu ambiente por meio do desenho conceitual, planejamento formal e posicionamento competitivo. Outras perspectivas descrevem o mesmo processo como visionário (Escola Empreendedora), mental (Escola Cognitiva), emergente (Escola da Aprendizagem), de conflito e poder (Escola Política), ideológico (Escola Cultural), ou, ainda, como um processo passivo (Escola Ambiental). Em sua proposição, esses autores sugerem que a formação de estratégia é um processo episódico que depende de condições temporais e espaciais da empresa, no sentido de que existem diferentes configurações de formação da estratégia (Escola da Configuração).

Whittington (1993) identificou quatro abordagens para a formação de estratégia. A Clássica entende que a formação de estratégia é um processo racional de análise deliberada com o objetivo de maximizar a vantagem em longo prazo da empresa. A Evolucionista sugere que mudanças ambientais são consideradas impossíveis de prever, e escolhas gerenciais não têm nenhuma influência nos destinos da organização, sendo que o mercado é que seleciona os mais aptos a sobreviverem. A Processualista reconhece a existência de limitações cognitivas que 
dificultam a elaboração de planos, e assim estratégias não ótimas emergem de um padrão de decisões passadas. E, por fim, a Sistêmica, que aponta para a relação de dependência entre a formação de estratégia e os sistemas sociais particulares nos quais ela surge.

Para Bailey e Avery (1998), o processo de desenvolvimento da estratégia não pode ser sempre caracterizado como intencional eplanejado. Em concordância com Whittington (1993) e Mintzberg, Ahlstrand e Lampel (2000, p. 182), esses autores afirmam que a estratégia pode surgir sob o efeito de diferentes influências. No seu modelo, eles adotam seis dimensões:

- planejamento: a estratégia é desenvolvida por meio de um processo de planejamento analítico, intencional e sequencial;

- incremental: a estratégia é desenvolvida de maneira evolucionária e com propósito, por meio de um processo iterativo de tentativa e erro;

- cultural: a estratégia é dirigida e guiada por aspectos culturais da organização, sua história e pressupostos e crenças compartilhadas entre seus membros;

- política: a estratégia é desenvolvida por meio de barganhas, negociação e influ- ência entre grupos de interesse internos à organização;

- comando: a estratégia é definida e determinada por um indivíduo poderoso dentro da organização;

- escolha forçada: a estratégia resulta de pressões externas que limitam a habilidade da organização em escolher sua direção estratégica.

As dimensões presentes na proposta de Bailey e Avery (1998) apresentam similaridades com outros modelos. Por exemplo, a dimensão planejamento se assemelha às proposições das Escolas do Design, Planejamento Posicionamento de Mintzberg, Ahlstrand eLampel(2000). De igual forma, a dimensão incremental e a Escola de Aprendizagem possuem aspectos comuns, assim como as dimensões cultural, política, comando e escolha forçada podem ser relacionadas às Escolas Cultural, Política, Empreendedora e Ambiente do esquema de Mintzberg, Ahlstrand e Lampel (2000). Cada uma dessas dimensões apresenta um conjunto particular de características que elucidam mais detalhadamente suas influências sobre o processo de desenvolvimento da estratégia, as quais são sintetizadas no Quadro 1.

Quadro 1 - Características das seis dimensões do desenvolvimento da estratégia

(Continua)

\section{DIMENSÃO CARACTERÍSTICAS}

As estratégias são o resultado de procedimentos racionais, metódicos e planejados sequencialmente Os objetivos estratégicos são escolhidos por executivos seniores

Planejamento A organização e o ambiente são analisados

Objetivos precisos e claros são definidos

Planos precisos de implantação são desenvolvidos

A estratégia é explicitada na forma de planos detalhados

A estratégia é continuamente ajustada às mudanças no ambiente operacional Opções estratégicas são continuamente avaliadas para ajustes

Incremental Comprometimento inicial com uma estratégia é sempre tentativo e sujeito à revisão A estratégia se desenvolve por meio de experimentação e implantação gradual Opções bem-sucedidas recebem recursos adicionais

A estratégia se desenvolve por meio de mudanças de pequena escala

Uma 'maneira de fazer as coisas' na organização influencia na direção estratégica As estratégias evoluem em acordo com um conjunto de pressupostos compartillados na organização

Cultural Um conjunto central de pressupostos compartilhados e baseados na experiência passada e história da empresa guia as ações estratégicas

A história organizacional direciona a busca e a seleção de opções estratégicas A estratégia não ajustada à cultura organizacional sofre resistência 
Quadro 1 - Características das seis dimensões do desenvolvimento da estratégia

(Conclusão)

\begin{tabular}{ll}
\hline & As estratégias são desenvolvidas por meio de negociação e barganha entre grupos \\
& Os grupos de interesse buscam realizar seus próprios objetivos desejados \\
Política & A influência na formação da estratégia aumenta com o poder \\
& O poder surge da habilidade de criar ou controlar o fluxo de recursos escassos \\
& O controle e fornecimento de informações também é uma fonte de poder \\
A estratégia desenvolvida é a aceitável para os grupos de interesse mais poderosos & \\
Um indivíduo é a força diretiva por trás de uma estratégia da organização & A estratégia é primariamente associada com o poder institucional de um indivíduo ou pequeno grupo \\
& A estratégia representa as aspirações deste indivíduo sobre o futuro da organização \\
& Um indivíduo tem um alto grau de controle sobre a estratégia \\
Escolha & As estratégias são prescritas pelo ambiente operacional \\
forçada & A escolha estratégica é restringida por forças externas que uma organização não é capaz de controlar \\
& A mudança estratégica é instigada por fontes externas à organização \\
& As organizações não são capazes de influenciar seu ambiente operacional \\
& Barreiras no ambiente restringem severamente a mobilidade estratégica
\end{tabular}

Fonte: Adaptado de BAILEY; AVERY, 1998, p. 183.

A partir dessa discussão conceitual, percebe-se um alinhamento entre diferentes modelos que procuram explicar o processo de formação de estratégia nas organizações.

Os estudos empíricos no campo da estratégia organizacional focam em geral dois tipos de resultados: conteúdo e processo estratégico.As investigações sobre o conteúdo estratégico centramse na identificação de alternativas de estratégias competitivas que são adotadas pelas organizações. Por sua vez, estudos sobre processo estratégico estão preocupados com o entendimento da forma como surge a estratégia nas organizações. $\mathrm{O}$ foco da pesquisa aqui relatada é do segundo tipo e, para contextualizar os resultados que serão apresentados na quarta seção do artigo, apresenta-se, a seguir, uma síntese de alguns estudos que tiveram foco semelhante. Por limitações de espaço, não é possível ser exaustivo nessa revisão. Dessa forma, optou-se em apresentar estudos que foram julgados representativos de diferentes abordagens no campo do conhecimento. Igualmente, para fins de comparação, buscou-se localizar estudos no setor hoteleiro que tivessem abordado a formação de estratégia em empresas de pequeno porte.

Rossetto et al. (2006) relatam os resultados de estudo sobre comportamento estratégico de dirigentes de pousadas localizadas em Florianópolis. A amostra compreendeu cinco empreendimentos com mais de dez anos de existência, em uma abordagem de multicasos. Embora os autores tenham olhado para outros aspectos de conteúdo estratégico em sua pesquisa, o resultado mais relevante para o propósito de nossa pesquisa se refere aos modos predominantes de formação de estratégia nas pousadas analisadas. Os resultados indicaram que houve uma predominância de processo adaptativo de formação de estratégia (três casos) e empreendedor (dois casos), conforme modelo de Mintzberg (1973). Ademais, em nenhum dos casos estudados foi identificado o modo de planejamento.

Para Mintzberg (1973), as características básicas desses processos são:

a) no modo empreendedor há uma ativa busca por oportunidades, o poder é centralizado no principal dirigente da organização, a formação da estratégia se dá em mudanças significativa orientadas pela busca do crescimento;

b) no modo adaptativo de formação de estratégia as metas não são muito claras e são altamente baseadas em reações a problemas ou ameaças;

c) no modo de planejamento há uma presença forte de analistas que avaliam de forma antecipada vantagens e 
desvantagens de alternativas de ação, procurando na implementação uma integração entre as decisões dos diferentes níveis hierárquicos da empresa. Apesar da limitação de número de casos analisados, este artigo apresenta indicações de que em pequenas empresas podem haver diferentes processos de formação de estratégia.

Lima (2007) também adotou a abordagem qualitativa do estudo de multicasos para investigar um aspecto específico da formação de estratégia: a construção de uma visão compartilhada por dirigentes de pequenas empresas. Sua análise focou sobre seis casos de empresas brasileiras e canadenses, em diferentes setores de atividades, divididos em dois grupos - empresas de base tecnológica e empresas tradicionais. Os resultados apresentados por Lima (2007) apontam para um papel fundamental do diálogo entre dirigentes de pequenas empresas na construção de uma visão estratégica para a empresa. Nesse diálogo, denominado de conversa estratégica por Lima (2007), percebe-se a importância do aprendizado contínuo na formação de estratégia por equipes de dirigentes de pequenas empresas. Depreende-se deste trabalho, também, que a complementaridade de papéis, funções e conhecimentos dos dirigentes exerce influência sobre a qualidade da conversa estratégica.

Santos, Alves e Almeida (2007) realizaram um estudo no centro-oeste mineiro dedicado a identificar os principais aspectos da formação de estratégia em micro e pequenas empresas, bem como verificar a associação de algumas características do processo de formação de estratégia com o desempenho. Em oposição aos dois estudos já apresentados, neste caso a abordagem de pesquisa foi quantitativa, com o uso de técnicas de análise de regressão linear múltipla e análise discriminante. Foram pesquisadas 50 empresas de diferentes setores de atividades, por meio da técnica de levantamento, com a aplicação de questionários respondidos pelo principal dirigente de cada empresa. Os resultados alcançados mostraram tendência das empresas da amostra a adotar processos de formação de estratégia deliberados e formais, estando o desempenho acima da média associado a uma estratégia de inovação e mudança.

Em uma abordagem qualitativa, também baseada em multicasos, Barros (2001) usou o Questionário de Desenvolvimento de Estratégia
(QDE) desenvolvido por Bailey e Avery (1998) para identificar o processo de formação de estratégias em empresas que atuam no setor de prestação de serviços para a internet. Neste estudo, o autor utiliza cinco das seis dimensões encontradas em Bailey e Avery (1998) - cultural, política, comando, escolha forçada eincremental. No entanto, a dimensão planejamento não é utilizada, sendo adotada uma dimensão denominada networking, que, em essência, sugere que a formação de estratégia se baseia em um processo de relações e consultas entre os participantes de uma rede de negócios na qual a empresa se insere. Não há menção no artigo de Barros (2001) sobre esta substituição, assim como não se encontra em Bailey e Avery (1998) referência a outra versão do QDE. Os resultados do estudo de Barros (2001) apontaram para uma predominância da dimensão incremental em três das empresas estudadas, sendo que em uma delas há também influência da dimensão networking. $\mathrm{Na}$ quarta empresa, a dimensão predominante foi o comando. Mais uma vez, pode-se perceber com base nesse estudo a diversidade de processos de formação de estratégia em pequenas empresas.

Um trabalho fundamental para o entendimento da formação de estratégias em pequenas empresas é o de Miller (1987a). Nesse texto são apresentados os resultados de estudo com 97 pequenas empresas canadenses que responderam a um questionário com medidas sobre estrutura e processo estratégico. O estudo evidenciou uma relação positiva entre aspectos do processo de formação de estratégia e estrutura, em especial o fato de que procedimentos sistemáticos de planejamento, análise ambiental, explicitação da estratégia e tomada de decisão eram mais frequentes em empresas com estruturas mais formais e integradas. Ao mesmo tempo, essa relação se mostrou mais forte nas empresas com melhor desempenho e maior ênfase em inovação. A contribuição principal desse estudo está relacionada às evidências de que a formação de estratégia em pequenas empresas varia conforme condições ambientais e estruturais, base da abordagem das configurações que foi proposta por Miller (1987b).

Em uma vertente complementar ao estudo de Miller anteriormente mencionado, Gibbons e O'Connor (2005) estudaram a relação entre características organizacionais e do estrategista sobre o processo de formação de estratégia em pequenas empresas. Com uma abordagem quantitativa, baseada em respostas obtidas com 359 empresas irlandesas, 
o estudo revelou que empresas empreendedoras tendem a adotar processos mais formais de planejamento estratégico, enquanto que empresas conservadoras se direcionam para abordagens incrementais de formação de estratégia. Empresas empreendedoras, neste estudo, são aquelas cuja estratégia revela uma forte ênfase na inovação, enquanto que empresas conservadoras observam a busca de eficiência e menores custos em seu comportamento estratégico. Outro resultado significativo foi a associação entre experiência do gestor da pequena empresa e preferência por processos menos formais de formação de estratégia. As contribuições dessa pesquisa apontam para uma combinação entre fatores organizacionais e individuais na escolha da abordagem que será adotada para o desenvolvimento estratégico.

Adotando um foco em empresas familiares com crescimento rápido, o trabalho apresentado por Upton, Teal e Felan (2001) revelou que 46 entre 65 empresas industriais familiares estadunidenses adotam planos estratégicos formais para a condução de seus negócios, dos quais mais da metade com um horizonte de planejamento acima de três anos. Por outro lado, as 19 empresas restantes da amostra divulgam apenas oralmente sua visão e missão. A amostra foi composta por empresas cuja taxa média de crescimento em vendas anual foi de $92,5 \%$, sendo que $55 \%$ das empresas apresentaram taxas acima de $50 \%$. Além disso, os resultados do estudo mostraram que essas empresas usam estratégias de alta qualidade e pioneirismo em seus mercados. Dessa forma, o estudo sugere que altas taxas de crescimento podem exigir práticas mais formais de formação de estratégia.

Os resultados do trabalho apresentado por Covin, Green e Slevin (2006) apontam os efeitos que práticas de formação de estratégia têm sobre a relação entre orientação empreendedora da empresa e seu desempenho. Orientação empreendedora é um conceito que revela a preocupação estratégica com o crescimento e inovação. Neste estudo, houve a preocupação de verificar como o impacto da orientação empreendedora poderia ser influenciado pelo modo de formação de estratégia, pela capacidade de aprendizagem estratégica a partir de fracassos e pela maior participação dos colaboradores no desenvolvimento da estratégia. Dados coletados com 110 pequenas empresas industriais localizadas em três Estados norte-americanos demonstraram que há uma relação positiva entre orientação empreendedora e crescimento de vendas. Além disso, essa relação se mostrou mais forte em empresas em que a formação de estratégia se dá por processos emergentes mais centralizados. Por outro lado, a capacidade de aprendizagem estratégica a partir de experiências malsucedidas não evidenciou efeito nessa relação.

Esse conjunto de trabalhos aponta para uma diversidade de processos de formação de estratégia em pequenas empresas, indicando que não há uma forma única ou correta de proceder. Há situações em que processos mais formalizados parecem predominar, em outros contextos a formação da estratégia por processos emergentes ou incrementais predomina, e há também situações de processos altamente informais conduzindo escolhas estratégicas. Em face da complexidade desse fenômeno, optou-se por realizar um estudo em um contexto específico - pequenos hotéis independentes - para verificar quais seriam as dimensões predominantes da formação de estratégia. $\mathrm{Na}$ próxima seção são descritos os procedimentos adotados para a realização desse estudo.

\section{PROCEDIMENTOS DA PESQUISA}

Para realizar este estudo foi feita a coleta de dados com uma amostra não aleatória de 18 pequenos hotéis localizados na cidade de Curitiba, PR. Os hotéis foram identificados com base na relação de hotéis disponíveis no catálogo telefônico da cidade. Os dados foram coletados a partir da aplicação de um questionário, em contato pessoal com o principal dirigente de cada empresa visitada. Nesseinstrumento de coleta de dados havia dois conjuntos de questões. Oprimeiro bloco levantou informações demográficas sobre a empresa e seu dirigente. O segundo bloco foi composto pelo Questionário de Desenvolvimento de Estratégia (QDE) proposto por Bailey e Avery (1998), que foi traduzido do original em inglês por um dos autores da pesquisa.

O QDE é composto por 36 afirmativas que correspondem às seis diferentes dimensões presentes no processo de formação de estratégia discutido na seção anterior. Os respondentes foram instruídos a assinalar para cada um dos itens, em uma escala de 7 pontos, ograu de correspondência da afirmativa com sua empresa, conforme a seguinte escala: 1 - você discorda fortemente da afirmação em relação a sua empresa; e 7 - você concorda fortemente com a afirmação em relação a sua empresa. No Quadro 2, apresentam-se alguns exemplos das afirmações que constam do QDE. 
Quadro 2 - Exemplos de itens que compõem o QDE

\begin{tabular}{|c|c|}
\hline DIMENSÃO & ITENS SELECIONADOS \\
\hline Planejamento & $\begin{array}{l}\text { Nós temos objetivos estratégicos precisos e definidos } \\
\text { Nós avaliamos opções estratégicas potenciais em relação aos objetivos estratégicos } \\
\text { formalmente definidos }\end{array}$ \\
\hline Incremental & $\begin{array}{l}\text { Nós nos comprometemos com uma estratégia, embora ela possa ser revista } \\
\text { Nós tendemos a desenvolver a estratégia experimentando e testando novas abordagens no mercado }\end{array}$ \\
\hline Cultural & $\begin{array}{l}\text { Há convicções e suposições sobre o modo de fazer as coisas que são típicas desta empresa } \\
\text { Há resistência a qualquer mudança estratégica que não combine com a nossa cultura }\end{array}$ \\
\hline Política & $\begin{array}{l}\text { Nossa direção estratégica é determinada por pessoas ou grupos poderosos } \\
\text { Nossa estratégia se desenvolve por um processo de negociação entre grupos ou indivíduos }\end{array}$ \\
\hline Comando & $\begin{array}{l}\text { O executivo principal tende a impor decisões estratégicas (em vez de consultar a equipe da } \\
\text { alta administração) } \\
\text { Nossa estratégia está intimamente associada a uma única pessoa }\end{array}$ \\
\hline Escolha forçada & $\begin{array}{l}\text { Forças externas da empresa determinam nossa direção estratégica } \\
\text { Existem obstáculos em nosso ambiente empresarial que limitam significativamente as } \\
\text { estratégias que podemos seguir }\end{array}$ \\
\hline
\end{tabular}

Fonte: Dados da pesquisa, 2009.

As instruções de preenchimento eram precedidas pelo seguinte texto, que o respondente era solicitado a ler:

Este instrumento considera o processo pelo qual a estratégia é desenvolvida dentro das empresas. É projetado para descobrir suas percepções de como são tomadas decisões estratégicas em sua empresa. Consideramos como decisões estratégicas aquelas que são caracterizadas por um grande comprometimento de recursos e lidam comassuntos de significativa importância para a empresa normalmente com impacto ou importância a um prazo mais longo do que curto; elas normalmente envolvem mais de uma função e envolvem mudança significativa.

As respostas foram tabuladas seguindo esquema proposto por Bailey e Avery (1998). No QDE, para cada uma das dimensões existem seis afirmativas. Dessa forma, a tabulação prevê um resultado que pode variar entre 6 e 42 para cada dimensão. Para facilitar uma visualização gráfica, os autores propuseram um mecanismo de redimensionamento da escala, com a subtração de 24 pontos do resultado em cada dimensão, o que faz com que o resultado final fique entre -18 e 18. Quanto mais positivo o resultado, mais predominante é a dimensão no processo de formação de estratégia. Quanto mais negativo, menos presente está a dimensão no processo de formação de estratégia. O instrumento de pesquisa completo e as instruções de tabulação podem ser obtidos com os autores do trabalho.

Com o objetivo de descrever quais sãos as dimensões mais presentes no processo de formação de estratégia nas empresas pesquisadas, apresentase na próxima seção os resultados do estudo. Em função do tipo de amostra e de seu tamanho reduzido, deve-se observar que os resultados do estudo não podem ser extrapolados para a população de pequenos hotéis de Curitiba, PR.

\section{DESCRIÇÃO E ANÁLISE DOS RESULTADOS}

Os dados obtidos nesta pesquisa permitem apresentar um perfil das características das empresas pesquisadas e dos seus dirigentes. Em seguida, os resultados da tabulação do QDE demonstram as dimensões mais presentes no processo de formação de estratégia.

A amostra que fez parte deste estudo foi composta por 18 hotéis independentes da cidade de Curitiba, PR. A média de vida dessas empresas é de 20,2 anos, com um desvio-padrão de 10,3. Na amostra há apenas uma empresa com menos de cinco anos de idade, e o restante das empresas existe há 
mais de doze anos. Esses dados indicam que todas as empresas já se consolidaram no mercado e podem ser consideradas competidores estáveis no mercado.

Em termos de número de empregados, a média da amostra foi de 25 trabalhadores com um desvio-padrão de 27,5 . Há uma variação muito grande no número de empregados na amostra, sendo o mínimo 3 e o máximo 88. Dezesseis das empresas foram classificadas pelos seus gestores como familiares.

Os gestores que responderam ao questionário têm uma experiência média no setor de 11,9 anos, com desvio-padrão de 8,4. Dos respondentes, cinco são mulheres. O perfil dos respondentes indica uma predominância do gênero masculino com idade média de 46,5 anos e mais de uma década de experiência no setor.

Por fim, o resultado da aplicação do QDE indicou que existe uma predominância de três dimensões no processo de formação de estratégia nos pequenos hotéis investigados. Em primeiro lugar, surge a dimensão incremental com uma pontuação média de 8,9 no QDE, o que indica 49,4\% do máximo de pontuação possível. Em segundo lugar, está a dimensão cultural com uma pontuação de 6,7, correspondendo a $37,2 \%$ do máximo possível. E, por fim, a dimensão planejamento, com 5,9 pontos, ou seja, 32,8\% dos pontos possíveis. O Gráfico 1 apresenta a representação desse resultado.

Gráfico 1 - Dimensões do processo de formação de estratégia

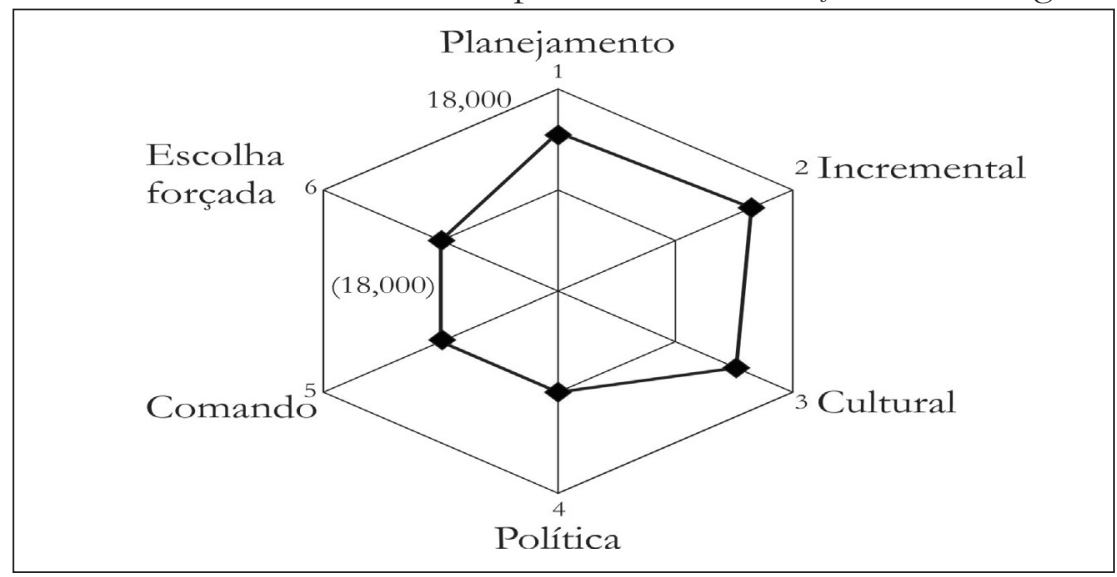

Fonte: Dados da pesquias, 2009.

Nesse gráfico, pode-se observar também que as dimensões política, comando e escolha forçada têm um impacto muito pequeno na formação de estratégia das empresas da amostra. A pontuação média nas três dimensões ficou em 0,11 para a dimensão política, e negativa para as outras duas.
Ao se verificar os dados para cada empresa, observou-se que esta combinação de dimensões presentes no processo de formação de estratégia ocorreu em 12 casos. Dessa forma, essa conjugação de dimensões parece ser a dominante na amostra estudada, estando presente em dois terços das empresas. As dimensões presentes em cada caso estudado são apresentadas no Quadro 3.

Quadro 3 - Dimensões presentes nos hotéis estudados

(Continua)

\begin{tabular}{clll}
\hline CASO & $\mathbf{1}^{\mathbf{a}}$ DIMENSÃO & $\mathbf{2}^{\mathbf{a}}$ DIMENSÃO & $\mathbf{3}^{\mathbf{a}}$ DIMENSÃO \\
\hline 01 & Planejamento & Incremental & Cultural \\
02 & Incremental & Cultural & \\
03 & Planejamento & Cultural & \\
04 & Incremental & Cultural & \\
05 & Incremental & Planejamento & Cultural \\
06 & Incremental & Planejamento & Cultural \\
\hline
\end{tabular}


Quadro 3 - Dimensões presentes nos hotéis estudados

\begin{tabular}{llll} 
& & & \\
(Conclusão) \\
08 & Incremental & Planejamento & Cultural \\
09 & Cultural & Incremental & Planejamento \\
10 & Incremental & & \\
11 & Incremental & Planejamento & Cultural \\
12 & Incremental & Planejamento & Cultural \\
13 & Incremental & Planejamento & Cultural \\
14 & Incremental & Cultural & Planejamento \\
15 & Cultural & Cultural & Planejamento \\
16 & Incremental & Incremental & Planejamento \\
17 & Incremental & Cultural & Planejamento \\
18 & Incremental & & \\
\hline
\end{tabular}

Fonte: Dados da pesquisa, 2009.

Estes resultados se assemelham aos obtidos por Rossetto et al. (2006) no estudo que realizaram com pousadas em Santa Catarina. De igual forma, o estudo de Barros (2001) demonstrou que a dimensão incremental foi a mais presente nas empresas de internet. No estudo de Upton, Tela e Felan (2001) observou-se a importância do planejamento para empresas de crescimento rápido. Embora não possamos afirmar que seja essa a situação dos hotéis pesquisados, pode-se dizer que a dimensão planejamento parece ser relevante para a longevidade das empresas. Por fim, Gibbons e O’Connor (2005) evidenciaram que empresas tradicionais tendem a processos incrementais de formação de estratégia. As empresas dessa amostra podem ser consideradas tradicionais, pois estão presentes no mercado há décadas.

Por outro lado, é interessante observar que em nenhum dos casos investigados houve o surgimento da dimensão comando. Este tipo de dimensão é muito frequente em pequenas empresas, nas quais a presença de um dirigente fundador exerce muita influência sobre o processo e as escolhas estratégica da organização. No entanto, no caso dessa amostra, pode-se argumentar que essas empresas, tendo em vista sua longa existência, já passaram o estágio de alta dependência das decisões do fundador e se configuram como organizações mais estáveis, nas quais os processos estratégicos são mais compartilhados.

\section{CONSIDERAÇÕES FINAIS}

O objetivo desse trabalho foi descrever as dimensões predominantes no processo de formação de estratégia de pequenos hotéis. A partir de um modelo conceitual que considera seis diferentes dimensões, os dados revelaram que nas pequenas empresas estudadas há a predominância do incrementalismo, ou seja, processos emergentes de formação de estratégia, combinado com aspectos de planejamento e culturais.

Essa combinação de dimensões pode ser explicada pelas características das empresas estudadas. Elas são pequenas empresas familiares que possuem uma tradição cultural marcada pela atuação há mais de uma década no mercado. Ao mesmo tempo, a natureza da atividade hoteleira demanda um conjunto de procedimentos sistematizados em sua operação diária, o que facilita a adoção de ferramentas de planejamento. Por fim, a necessidade de enfrentar um mercado cada vez mais competitivo leva à adoção de mudanças estratégicas incrementais que respondam às transformações percebidas no mercado.

Em suma, o presente estudo ajuda a compreender as nuances do processo de formação de estratégia em pequenas empresas, trazendo novas evidências que demonstram a possibilidade de configurações de dimensões na formação de estratégia nesse contexto. Os resultados do presente estudo são consistentes com estudos anteriores e ajudam a consolidar o conhecimento sobre o tema. 
Estudos futuros podem avançar no sentido de explorar quais são as possíveis relações entre características organizacionais, processos de formação de estratégia, estratégia competitiva e desempenho da empresa. Esse conhecimento, ainda em formação no campo daadministração estratégica, pode revelarcomo diferentes configurações dessas dimensões podem levar a desempenho bem-sucedido em diferentes mercados.

\section{REFERÊNCIAS}

BAILEY, A.; AVERY, C. Discovering and defining the process of strategy development. In: AMBROSINI, V.; JOHNSON, G.; SCHOLES, K. (Org.). Exploring techniques of analysis and evaluation in strategic management. Harlow: Prentice-Hall, 1998. p. 181-201.

BARROS, M. A. Estratégia nas empresas da Internet: lições da informalidade. Revista de Administração de Empresas, v. 41, n. 4, p. 32-43, 2001.

COOPER, A. C. Strategic management: new ventures and small business. Long Range Planning, v. 14, n. 5, p. 39-45, 1981.

COVIN, J. G.; GREEN, K. M.; SLEVIN, D. P. Strategic process effects on the entrepreneurial orientation-sales growth rate relationship. Entrepreneurship Theory and Practice, v. 30, n. 1, p. 56-81, 2006.

D'AMBOISE, G.;MULDOWNEY,M.Management theory for small business: attempts and requirements. Academy of Management Review, v. 13, n. 2, p. 226-240, 1988.

GIBBONS, P. T.; O'CONNOR, T. Influences on strategic planning processes among Irish SMEs. Journal of Small Business Management, v. 43, n. 2, p. 170-186, 2005.

HITT, M. A. et al. Strategic entrepreneurship: creating a new mindset. 2nd ed. Oxford: Blackwell, 2002.

HOCAYEN-DA-SILVA, A.J.;TEIXEIRA, R. T. Ambiente competitivo e vantagem competitiva a partir de uma abordagem integradora: estudo de caso no setor hoteleiro em Curitiba. Turismo: Visão e Ação, v. 9, n. 1, p. 19-35, 2007.

JENKINS, M.; AMBROSINI, V. Strategic management: a multi-perspective approach. 2nd ed. New York: Palgrave, 2002.

LIMA, E. Visão compartilhada equipe de direção e gestão estratégica de pequenas e médias empresas: um estudo multi-caso e internacional. Revista de Negócios, v. 12, n. 4, p. 86-100, 2007.
MILLER, D. Strategy making and structure: analysis and implications for performance. Academy of Management Journal, v. 30, n. 1, p. 7-32, 1987a.

MILLER, D. The genesis of configuration. Academy of Management Review, v. 12, n. 4, p. 686-701, $1987 \mathrm{~b}$.

MINTZBERG, H. Strategy-making in the three modes. California Management Review, v. 16, n. 2, p. 44-53, 1973.

MINTZBERG,H.;AHLSTRAND, B.;LAMPEL, J. Safari de estratégia: um roteiro pela selva do planejamento estratégico. 2. ed. Porto Alegre: Bookman, 2000.

MINTZBERG, H.; QUINN, J. B. O processo da estratégia. 3. ed. Porto Alegre: Bookman, 2001.

ROBINSON Jr., R. B.; PEARCE II, J. A. Research thrusts in small firm strategic planning. Academy of Management Review, v. 9, n. 1, p. 128-137, 1984.

ROSSETTO, C. R. et al. Comportamento estratégico em pequenas e médias empresas: estudo multi-casos em pousadas na Lagoa da Conceição - Florianópolis - SC. Revista de Negócios, v. 11, n. 3, p. 93-108, 2006.

SANTOS, L. L. da S.; ALVES, R. C.; ALMEIDA, K. N. T. Formação da estratégia em micro e pequenas empresas: um estudo no centro-oeste mineiro. Revista de Administração de Empresas, v. 44, n. 4, p. 59-73, 2007.

UPTON, N.; TEAL, E. J.; FELAN, J. T. Strategic and business plan practices of fast growth family firms. Journal of Small Business Management, v. 39, n. 1, p. 60-72, 2001.

WHITTINGTON, R. What is strategy and does it matter? 2nd ed. London: Routledge, 1993.

Recebido: 17/08/2008

Received: 08/17/2008

Aprovado: 22/09/2008

Approved: 09/22/2008 